Acta Crystallographica Section E

Structure Reports

Online

ISSN 1600-5368

\section{(Z)-N-tert-Butyl-2-(4-methoxyanilino)- $N^{\prime}$-(4-methoxyphenyl)-2-phenyl- acetimidamide}

\section{Sue A. Roberts, ${ }^{a *}$ Biswajit Saha, ${ }^{b, c}$ Brendan Frett $^{b, c}$ and Hong-Yu Li $\mathbf{i}^{\mathrm{b}, \mathrm{c}}$}

\begin{abstract}
${ }^{a}$ Department of Chemistry and Biochemistry, 1306 E University Boulevard, The University of Arizona, Tucson, AZ 85721, USA, 'bCollege of Pharmacy, Department of Pharmacology and Toxicology, The University of Arizona, Tucson, AZ 85721, USA, and ${ }^{\mathrm{C}}$ Bio5 Oro Valley, The University of Arizona, Oro Valley, AZ 85737, USA Correspondence e-mail: suer@email.arizona.edu

Received 10 April 2013; accepted 9 May 2013

Key indicators: single-crystal X-ray study; $T=100 \mathrm{~K}$; mean $\sigma(\mathrm{C}-\mathrm{C})=0.002 \AA$; $R$ factor $=0.043 ; w R$ factor $=0.106 ;$ data-to-parameter ratio $=22.9$.
\end{abstract}

In the crystal of the title compound, $\mathrm{C}_{26} \mathrm{H}_{31} \mathrm{~N}_{3} \mathrm{O}_{2}$, pairs of $\mathrm{N}-$ $\mathrm{H}$... O hydrogen bonds link molecules, forming inversion dimers, which enclose an $R_{2}^{2}(20)$ ring motif. One $\mathrm{N}$ atom does not form hydrogen bonds and lies in a hydrophobic pocket with closest intermolecular contacts of $4.196(2)$ and 4.262 (2) ̊..

\section{Related literature}

For the synthesis of the title compound, and a discussion of the use of the three-component Ugi reaction to synthesize amidines, see: Saha et al. (2013).

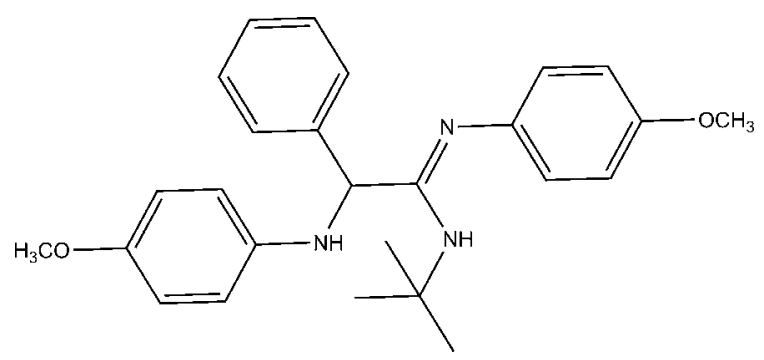

\section{Experimental}

Crystal data

$\mathrm{C}_{26} \mathrm{H}_{31} \mathrm{~N}_{3} \mathrm{O}_{2}$

$M_{r}=417.54$

Triclinic, $P \overline{1}$

$a=10.0804(17) \AA$ $b=10.5784$ (17) $\AA$

$c=11.1573(18) \AA$

$\alpha=80.982(4)^{\circ}$

$\beta=85.152(4)^{\circ}$

$\gamma=80.270(4)^{\circ}$

$V=1156.1(3) \AA^{3}$

$Z=2$

Mo $K \alpha$ radiation

$\mu=0.08 \mathrm{~mm}^{-1}$

$T=100 \mathrm{~K}$

$0.20 \times 0.20 \times 0.10 \mathrm{~mm}$

Data collection

Bruker Kappa APEXII DUO CCD diffractometer

Absorption correction: multi-scan

(SADABS; Bruker, 2009)

$T_{\text {min }}=0.89, T_{\max }=0.99$

Refinement

$R\left[F^{2}>2 \sigma\left(F^{2}\right)\right]=0.043$

$w R\left(F^{2}\right)=0.106$

$S=0.94$

6607 reflections

289 parameters

32612 measured reflections 6607 independent reflections 5476 reflections with $I>2 \sigma(I)$

$R_{\text {int }}=0.019$

Table 1

Hydrogen-bond geometry $\left(\AA{ }^{\circ}\right)$.

\begin{tabular}{lllll}
\hline$D-\mathrm{H} \cdots A$ & $D-\mathrm{H}$ & $\mathrm{H} \cdots A$ & $D \cdots A$ & $D-\mathrm{H} \cdots A$ \\
\hline $\mathrm{N} 2-\mathrm{H} 2 \cdots \mathrm{O}^{\mathrm{i}}$ & $0.887(15)$ & $2.328(15)$ & $3.1698(13)$ & $158.5(12)$ \\
\hline
\end{tabular}

Symmetry code: (i) $-x+1,-y+2,-z+2$.

Data collection: APEX2 (Bruker, 2009); cell refinement: SAINT (Bruker, 2009); data reduction: $S A I N T$; $\operatorname{program}(\mathrm{s})$ used to solve structure: SHELXS97 (Sheldrick, 2008); program(s) used to refine structure: SHELXL97 (Sheldrick, 2008) and OLEX2 (Dolomanov et al., 2009); molecular graphics: ORTEP-3 for Windows (Farrugia, 2012) and Mercury (Macrae et al., 2006); software used to prepare material for publication: publCIF (Westrip, 2010).

The Bruker Kappa APEXII DUO was purchased with funding from NSF grant CHE-0741837. The work was supported by start-up funds from the University of Arizona to HYL.

Supplementary data and figures for this paper are available from the IUCr electronic archives (Reference: PK2477).

\section{References}

Bruker (2009). APEX2, SAINT and SADABS. Bruker AXS Inc., Madison, Wisconsin, USA.

Dolomanov, O. V., Bourhis, L. J., Gildea, R. J., Howard, J. A. K. \& Puschmann, H. (2009). J. Appl. Cryst. 42, 339-341.

Farrugia, L. J. (2012). J. Appl. Cryst. 45, 849-854.

Macrae, C. F., Edgington, P. R., McCabe, P., Pidcock, E., Shields, G. P., Taylor, R., Towler, M. \& van de Streek, J. (2006). J. Appl. Cryst. 39, 453-457.

Saha, B., Frett, B., Wang, Y. \& Li, H.-Y. (2013). Tetrahedron Lett. 54, 2340 2343.

Sheldrick, G. M. (2008). Acta Cryst. A64, 112-122.

Westrip, S. P. (2010). J. Appl. Cryst. 43, 920-925. 


\section{supporting information}

Acta Cryst. (2013). E69, o902 [doi:10.1107/S1600536813012877]

\section{(Z)-N-tert-Butyl-2-(4-methoxyanilino)-N'-(4-methoxyphenyl)-2-phenyl- acetimidamide}

\section{Sue A. Roberts, Biswajit Saha, Brendan Frett and Hong-Yu Li}

\section{S1. Comment}

The title compound was prepared as a secondary product of the three component Ugi reaction (Saha et al., 2013). The central $\mathrm{C} 1-\mathrm{C} 22$ single bond has four bulky substituents arranged, roughly, as if pointing at the corners of a tetrahedron. The angle between the planes containing the substituents, defined by $\mathrm{N} 1-\mathrm{C} 22-\mathrm{N} 3$ and $\mathrm{C} 2-\mathrm{C} 1-\mathrm{C} 20$, is $72.41(1)^{\circ}$. Pairs of hydrogen bonds between N2 and O1 [3.170 (1) $\AA]$ connect two molecules across an inversion center, creating a cavity around the inversion center with a diameter of about $5 \AA$. The closest contact across the inversion center involving atoms not involved in the hydrogen bonding is 3.151 (3) $\AA$ between the hydrogen bonded to $\mathrm{C} 3$ and its symmetry equivalent (symmetry transformation $-x+1,-y+2,-z+2$ ). The hydrogen bonding graph set is $\mathrm{R}_{2}^{2}(20)$. Nitrogen N3 is unusual in that, although protonated, it does not form hydrogen bonds with acceptor atoms. Instead, it lies in a hydrophobic cavity with closest intermolecular contacts of 4.196 (2) $\AA$ and 4.262 (2) $\AA$ to C15 and C16 respectively.

\section{S2. Experimental}

The compound was synthesized as previously reported [compound 5a in (Saha et al., 2013)]. The crude residue was purified by silica gel column chromatography using (10-30\%) ethylacetate-hexane to obtain the pure product. The pure compound was dissolved in 50\% ethylacetate-hexane and kept at room temperature for 2 days during which crystals formed.

\section{S3. Refinement}

All hydrogen atoms were visible in a difference Fourier map and, except for $\mathrm{H} 2$ were added at calculated positions. Bond distances are set to $0.95 \AA$ for aromatic carbon-hydrogen bonds, $0.98 \AA$ for methyl group carbon-hydrogen bonds and $0.88 \AA$ for nitrogen-hydrogen bonds. Thermal parameters for hydrogen atoms were set to 1.2 times the isotropic equivalent thermal parameter of the atom to which the hydrogen atom is bonded, except for methyl group hydrogen atoms where the thermal parameter was set to 1.5 times the isotropic equivalent thermal parameter of the carbon atom the hydrogen atom is bonded to. The positional and isotropic thermal parameters of $\mathrm{H} 2$ were refined. 


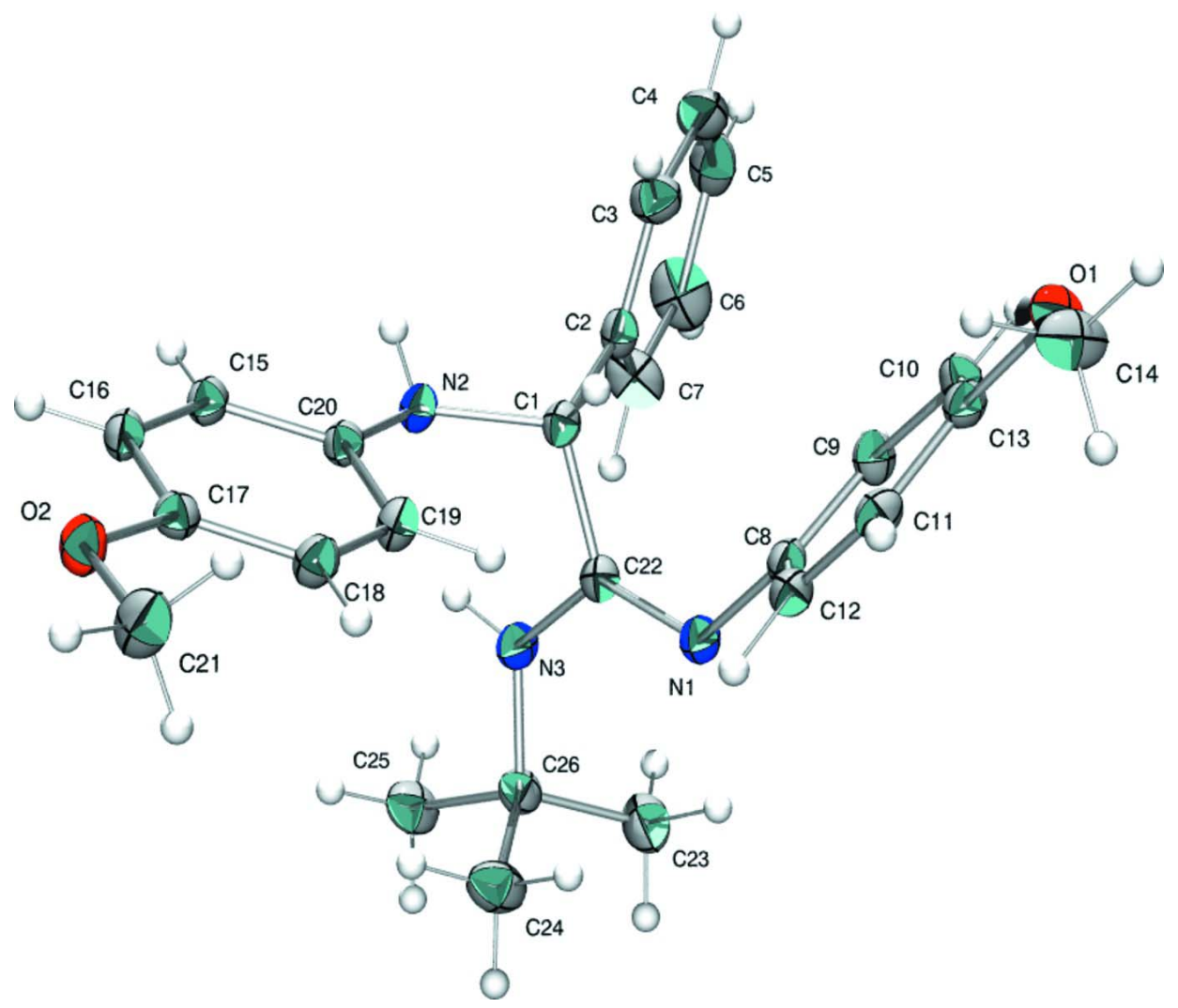

\section{Figure 1}

The molecular structure of the title compound. Non-hydrogen atoms are shown as $50 \%$ probability ellipsoids. 


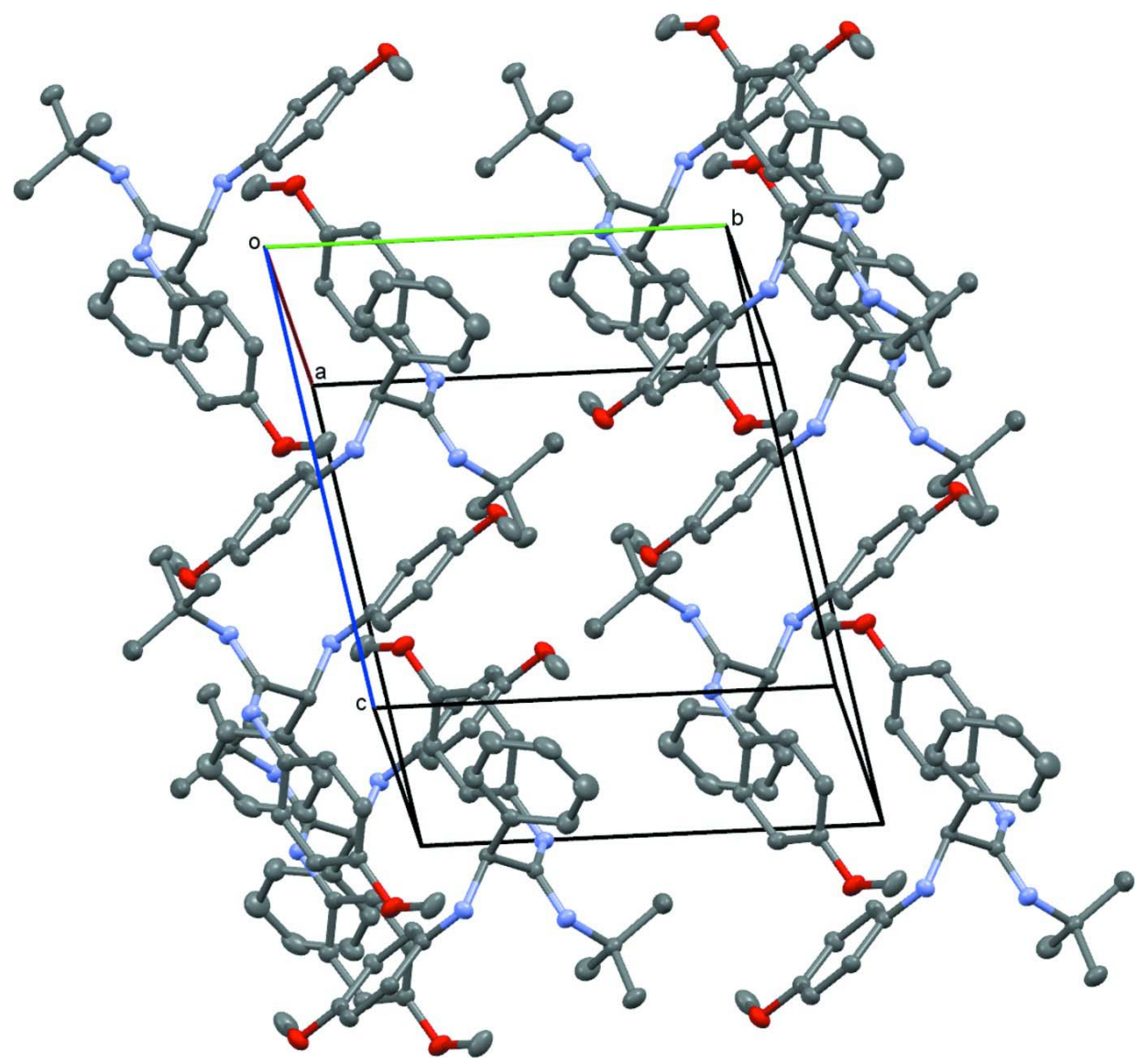

\section{Figure 2}

View showing unit cell packing. Unit cell axes are labeled in the figure.

\section{(Z)-N-tert-Butyl-2-(4-methoxyanilino)-N'-(4-methoxyphenyl)-2-phenylacetimidamide}

\section{Crystal data}

$\mathrm{C}_{26} \mathrm{H}_{31} \mathrm{~N}_{3} \mathrm{O}_{2}$

$M_{r}=417.54$

Triclinic, $P \overline{1}$

$a=10.0804(17) \AA$

$b=10.5784(17) \AA$

$c=11.1573(18) \AA$

$\alpha=80.982(4)^{\circ}$

$\beta=85.152(4)^{\circ}$

$\gamma=80.270(4)^{\circ}$

$V=1156.1(3) \AA^{3}$

\section{Data collection}

Bruker Kappa APEXII DUO CCD diffractometer

Radiation source: fine-focus sealed tube Graphite monochromator

Detector resolution: 8.3333 pixels $\mathrm{mm}^{-1}$ $\varphi$ and $\omega$ scans
$Z=2$

$F(000)=448$

$D_{\mathrm{x}}=1.199 \mathrm{Mg} \mathrm{m}^{-3}$

Mo $K \alpha$ radiation, $\lambda=0.71073 \AA$

Cell parameters from 9952 reflections

$\theta=2.9-29.8^{\circ}$

$\mu=0.08 \mathrm{~mm}^{-1}$

$T=100 \mathrm{~K}$

Block, colourless

$0.20 \times 0.20 \times 0.10 \mathrm{~mm}$

Absorption correction: multi-scan

(SADABS; Bruker, 2009)

$T_{\min }=0.89, T_{\max }=0.99$

32612 measured reflections

6607 independent reflections

5476 reflections with $I>2 \sigma(I)$

$R_{\text {int }}=0.019$ 
$\theta_{\text {max }}=29.9^{\circ}, \theta_{\text {min }}=1.9^{\circ}$

$h=-14 \rightarrow 11$

Refinement

Refinement on $F^{2}$

Least-squares matrix: full

$R\left[F^{2}>2 \sigma\left(F^{2}\right)\right]=0.043$

$w R\left(F^{2}\right)=0.106$

$S=0.94$

6607 reflections

289 parameters

0 restraints

Primary atom site location: structure-invariant

direct methods $k=-14 \rightarrow 12$

$l=-15 \rightarrow 13$

Secondary atom site location: difference Fourier map

Hydrogen site location: inferred from neighbouring sites

$\mathrm{H}$ atoms treated by a mixture of independent and constrained refinement

$w=1 /\left[\sigma^{2}\left(F_{\mathrm{o}}^{2}\right)+(0.0397 P)^{2}+0.6486 P\right]$ where $P=\left(F_{\mathrm{o}}^{2}+2 F_{\mathrm{c}}^{2}\right) / 3$

$(\Delta / \sigma)_{\max }=0.001$

$\Delta \rho_{\max }=0.36 \mathrm{e} \AA^{-3}$

$\Delta \rho_{\min }=-0.38$ e $\AA^{-3}$

\section{Special details}

Geometry. All e.s.d.'s (except the e.s.d. in the dihedral angle between two l.s. planes) are estimated using the full covariance matrix. The cell e.s.d.'s are taken into account individually in the estimation of e.s.d.'s in distances, angles and torsion angles; correlations between e.s.d.'s in cell parameters are only used when they are defined by crystal symmetry. An approximate (isotropic) treatment of cell e.s.d.'s is used for estimating e.s.d.'s involving 1.s. planes.

Refinement. Refinement of $F^{2}$ against ALL reflections. The weighted $R$-factor $w R$ and goodness of fit $S$ are based on $F^{2}$, conventional $R$-factors $R$ are based on $F$, with $F$ set to zero for negative $F^{2}$. The threshold expression of $F^{2}>\sigma\left(F^{2}\right)$ is used only for calculating $R$-factors(gt) etc. and is not relevant to the choice of reflections for refinement. $R$-factors based on $F^{2}$ are statistically about twice as large as those based on $F$, and $R$ - factors based on ALL data will be even larger.

Fractional atomic coordinates and isotropic or equivalent isotropic displacement parameters $\left(\AA^{2}\right)$

\begin{tabular}{lllll}
\hline & $x$ & $y$ & $z$ & $U_{\text {iso }} * U_{\text {eq }}$ \\
\hline C20 & $0.37947(10)$ & $1.03405(9)$ & $0.68022(9)$ & $0.01884(19)$ \\
C11 & $0.15660(10)$ & $0.92039(10)$ & $1.16518(9)$ & $0.0213(2)$ \\
H11 & 0.0822 & 0.9836 & 1.1842 & $0.026^{*}$ \\
C18 & $0.15655(11)$ & $1.15884(10)$ & $0.70473(10)$ & $0.0226(2)$ \\
H18 & 0.0708 & 1.1695 & 0.7475 & $0.027^{*}$ \\
C15 & $0.40664(11)$ & $1.12635(10)$ & $0.57996(9)$ & $0.02047(19)$ \\
H15 & 0.4919 & 1.1155 & 0.5363 & $0.025^{*}$ \\
C16 & $0.31125(11)$ & $1.23257(10)$ & $0.54406(9)$ & $0.0219(2)$ \\
H16 & 0.3313 & 1.2940 & 0.4761 & $0.026^{*}$ \\
C22 & $0.35777(10)$ & $0.75795(9)$ & $0.82814(9)$ & $0.01868(19)$ \\
C10 & $0.36795(11)$ & $0.78673(11)$ & $1.21611(9)$ & $0.0228(2)$ \\
H10 & 0.4377 & 0.7582 & 1.2709 & $0.027^{*}$ \\
C12 & $0.16277(10)$ & $0.86653(10)$ & $1.05826(9)$ & $0.0212(2)$ \\
H12 & 0.0906 & 0.8921 & 1.0057 & $0.025^{*}$ \\
C19 & $0.25281(11)$ & $1.05135(10)$ & $0.74063(10)$ & $0.0224(2)$ \\
H19 & 0.2315 & 0.9890 & 0.8074 & $0.027^{*}$ \\
C8 & $0.27219(10)$ & $0.77613(10)$ & $1.02648(9)$ & $0.01957(19)$ \\
C1 & $0.46163(10)$ & $0.84821(9)$ & $0.83240(9)$ & $0.01839(18)$ \\
H1 & 0.4260 & 0.9082 & 0.8927 & $0.022^{*}$ \\
C17 & $0.18548(10)$ & $1.25005(10)$ & $0.60709(9)$ & $0.0206(2)$ \\
C9 & $0.37371(11)$ & $0.73460(10)$ & $1.10887(9)$ & $0.0223(2)$ \\
H9 & 0.4473 & 0.6700 & 1.0910 & $0.027^{*}$ \\
& & & &
\end{tabular}




\begin{tabular}{|c|c|c|c|c|}
\hline $\mathrm{C} 2$ & $0.59808(10)$ & $0.77504(10)$ & $0.87123(9)$ & $0.0209(2)$ \\
\hline $\mathrm{C} 26$ & $0.27437(11)$ & $0.63246(10)$ & $0.68176(9)$ & $0.0220(2)$ \\
\hline $\mathrm{C} 3$ & $0.67295(12)$ & $0.83256(11)$ & $0.93977(11)$ & $0.0280(2)$ \\
\hline H3 & 0.6367 & 0.9144 & 0.9636 & $0.034 *$ \\
\hline $\mathrm{C} 24$ & $0.12887(12)$ & $0.70234(13)$ & $0.67994(12)$ & 0.0318 (3) \\
\hline $\mathrm{H} 24 \mathrm{~A}$ & 0.0962 & 0.7183 & 0.7623 & $0.048^{*}$ \\
\hline H24B & 0.0722 & 0.6484 & 0.6505 & $0.048^{*}$ \\
\hline $\mathrm{H} 24 \mathrm{C}$ & 0.1249 & 0.7852 & 0.6258 & $0.048^{*}$ \\
\hline $\mathrm{C} 7$ & $0.65156(13)$ & $0.65363(12)$ & $0.83952(12)$ & $0.0329(3)$ \\
\hline H7 & 0.6010 & 0.6121 & 0.7938 & $0.039 *$ \\
\hline $\mathrm{C} 25$ & $0.32629(13)$ & $0.61040(12)$ & $0.55297(10)$ & 0.0299 (2) \\
\hline $\mathrm{H} 25 \mathrm{~A}$ & 0.3167 & 0.6936 & 0.4991 & $0.045^{*}$ \\
\hline H25B & 0.2739 & 0.5524 & 0.5239 & $0.045^{*}$ \\
\hline $\mathrm{H} 25 \mathrm{C}$ & 0.4215 & 0.5710 & 0.5532 & $0.045^{*}$ \\
\hline C4 & $0.80027(13)$ & $0.77209(13)$ & $0.97403(12)$ & $0.0336(3)$ \\
\hline H4 & 0.8505 & 0.8127 & 1.0209 & $0.040^{*}$ \\
\hline $\mathrm{C} 21$ & $-0.02644(12)$ & $1.38154(12)$ & $0.63778(12)$ & $0.0333(3)$ \\
\hline $\mathrm{H} 21 \mathrm{~A}$ & -0.0784 & 1.3119 & 0.6344 & $0.050^{*}$ \\
\hline $\mathrm{H} 21 \mathrm{~B}$ & -0.0785 & 1.4649 & 0.6052 & $0.050^{*}$ \\
\hline $\mathrm{H} 21 \mathrm{C}$ & -0.0078 & 1.3829 & 0.7223 & $0.050 *$ \\
\hline $\mathrm{C} 14$ & $0.15403(12)$ & $1.02124(13)$ & $1.38420(12)$ & $0.0335(3)$ \\
\hline $\mathrm{H} 14 \mathrm{~A}$ & 0.1460 & 1.0989 & 1.3229 & $0.050 *$ \\
\hline H14B & 0.1674 & 1.0449 & 1.4633 & $0.050^{*}$ \\
\hline $\mathrm{H} 14 \mathrm{C}$ & 0.0715 & 0.9827 & 1.3897 & $0.050^{*}$ \\
\hline $\mathrm{C} 5$ & $0.85386(12)$ & $0.65322(13)$ & $0.94015(12)$ & $0.0350(3)$ \\
\hline H5 & 0.9419 & 0.6128 & 0.9616 & $0.042 *$ \\
\hline $\mathrm{C} 23$ & $0.28606(16)$ & $0.50314(12)$ & $0.76560(12)$ & $0.0366(3)$ \\
\hline $\mathrm{H} 23 \mathrm{~A}$ & 0.3800 & 0.4597 & 0.7629 & $0.055^{*}$ \\
\hline H23B & 0.2285 & 0.4483 & 0.7388 & $0.055^{*}$ \\
\hline $\mathrm{H} 23 \mathrm{C}$ & 0.2573 & 0.5183 & 0.8490 & $0.055^{*}$ \\
\hline C6 & $0.77862(14)$ & 0.59305 (14) & $0.87460(13)$ & $0.0405(3)$ \\
\hline H6 & 0.8141 & 0.5098 & 0.8535 & $0.049 *$ \\
\hline $\mathrm{O} 2$ & $0.09729(8)$ & $1.35968(7)$ & $0.56724(7)$ & $0.02582(17)$ \\
\hline $\mathrm{O} 1$ & $0.26631(8)$ & $0.92977(8)$ & $1.35034(7)$ & $0.02582(17)$ \\
\hline N2 & $0.47953(9)$ & $0.92663(8)$ & $0.71356(8)$ & $0.01953(17)$ \\
\hline N3 & $0.36308(9)$ & $0.71424(9)$ & $0.71966(8)$ & $0.02124(18)$ \\
\hline $\mathrm{H} 3 \mathrm{~A}$ & 0.4268 & 0.7374 & 0.6658 & $0.025^{*}$ \\
\hline N1 & $0.27336(9)$ & $0.72595(9)$ & $0.91628(8)$ & $0.02123(18)$ \\
\hline $\mathrm{C} 13$ & $0.26038(10)$ & $0.88065(10)$ & $1.24380(9)$ & $0.0204(2)$ \\
\hline $\mathrm{H} 2$ & $0.5625(15)$ & 0.9449 (14) & $0.6977(13)$ & $0.027(3)^{*}$ \\
\hline
\end{tabular}

Atomic displacement parameters $\left(\AA^{2}\right)$

\begin{tabular}{lllllll}
\hline & $U^{11}$ & $U^{22}$ & $U^{33}$ & $U^{12}$ & $U^{13}$ & $U^{23}$ \\
\hline C20 & $0.0210(4)$ & $0.0187(4)$ & $0.0171(4)$ & $-0.0043(4)$ & $-0.0002(3)$ & $-0.0025(3)$ \\
C11 & $0.0189(4)$ & $0.0221(5)$ & $0.0226(5)$ & $-0.0038(4)$ & $0.0033(4)$ & $-0.0041(4)$ \\
C18 & $0.0203(5)$ & $0.0226(5)$ & $0.0236(5)$ & $-0.0037(4)$ & $0.0019(4)$ & $-0.0008(4)$ \\
C15 & $0.0230(5)$ & $0.0221(5)$ & $0.0164(4)$ & $-0.0057(4)$ & $0.0020(4)$ & $-0.0025(3)$
\end{tabular}


supporting information

$\begin{array}{lllllll}\text { C16 } & 0.0275(5) & 0.0212(5) & 0.0168(4) & -0.0066(4) & 0.0000(4) & 0.0002(3) \\ \text { C22 } & 0.0206(4) & 0.0177(4) & 0.0170(4) & -0.0019(3) & -0.0004(3) & -0.0022(3) \\ \text { C10 } & 0.0227(5) & 0.0274(5) & 0.0177(4) & -0.0019(4) & -0.0007(4) & -0.0033(4) \\ \text { C12 } & 0.0198(4) & 0.0246(5) & 0.0191(4) & -0.0052(4) & 0.0003(4) & -0.0017(4) \\ \text { C19 } & 0.0229(5) & 0.0210(5) & 0.0212(5) & -0.0034(4) & 0.0025(4) & 0.0016(4) \\ \text { C8 } & 0.0219(5) & 0.0212(5) & 0.0160(4) & -0.0072(4) & 0.0026(4) & -0.0018(3) \\ \text { C1 } & 0.0202(4) & 0.0185(4) & 0.0160(4) & -0.0032(3) & 0.0014(3) & -0.0021(3) \\ \text { C17 } & 0.0228(5) & 0.0189(5) & 0.0205(4) & -0.0036(4) & -0.0036(4) & -0.0022(4) \\ \text { C9 } & 0.0231(5) & 0.0236(5) & 0.0186(4) & -0.0005(4) & 0.0014(4) & -0.0035(4) \\ \text { C2 } & 0.0204(4) & 0.0227(5) & 0.0176(4) & -0.0023(4) & 0.0017(4) & 0.0003(4) \\ \text { C26 } & 0.0253(5) & 0.0227(5) & 0.0190(4) & -0.0051(4) & -0.0019(4) & -0.0050(4) \\ \text { C3 } & 0.0265(5) & 0.0246(5) & 0.0331(6) & -0.0050(4) & -0.0047(4) & -0.0024(4) \\ \text { C24 } & 0.0237(5) & 0.0407(7) & 0.0322(6) & -0.0036(5) & -0.0011(4) & -0.0112(5) \\ \text { C7 } & 0.0319(6) & 0.0317(6) & 0.0344(6) & 0.0050(5) & -0.0052(5) & -0.0120(5) \\ \text { C25 } & 0.0323(6) & 0.0362(6) & 0.0235(5) & -0.0045(5) & -0.0005(4) & -0.0132(4) \\ \text { C4 } & 0.0283(6) & 0.0347(6) & 0.0371(6) & -0.0080(5) & -0.0088(5) & 0.0036(5) \\ \text { C21 } & 0.0233(5) & 0.0299(6) & 0.0406(7) & 0.0017(4) & -0.0003(5) & 0.0062(5) \\ \text { C14 } & 0.0255(5) & 0.0433(7) & 0.0359(6) & -0.0052(5) & 0.0066(5) & -0.0234(5) \\ \text { C5 } & 0.0249(5) & 0.0396(7) & 0.0336(6) & 0.0028(5) & -0.0021(5) & 0.0080(5) \\ \text { C23 } & 0.0594(8) & 0.0243(6) & 0.0291(6) & -0.0136(6) & -0.0126(6) & -0.0005(4) \\ \text { C6 } & 0.0363(7) & 0.0366(7) & 0.0433(7) & 0.0128(5) & -0.0028(6) & -0.0091(6) \\ \text { O2 } & 0.0252(4) & 0.0223(4) & 0.0267(4) & -0.0005(3) & -0.0026(3) & 0.0034(3) \\ \text { O1 } & 0.0240(4) & 0.0337(4) & 0.0215(4) & -0.0035(3) & 0.0026(3) & -0.0124(3) \\ \text { N2 } & 0.0194(4) & 0.0206(4) & 0.0172(4) & -0.0035(3) & 0.0028(3) & -0.0002(3) \\ \text { N3 } & 0.0237(4) & 0.0240(4) & 0.0171(4) & -0.0065(3) & 0.0030(3) & -0.0056(3) \\ \text { N1 } & 0.0246(4) & 0.0233(4) & 0.0164(4) & -0.0062(3) & 0.0010(3) & -0.0033(3) \\ \text { C13 } & 0.0213(5) & 0.0235(5) & 0.0173(4) & -0.0069(4) & 0.0035(4) & -0.0047(4) \\ & & & & & \end{array}$

Geometric parameters $\left(\AA,{ }^{o}\right)$

\begin{tabular}{llll}
\hline $\mathrm{C} 20-\mathrm{C} 15$ & $1.4057(14)$ & $\mathrm{C} 26-\mathrm{C} 25$ & $1.5264(15)$ \\
$\mathrm{C} 20-\mathrm{C} 19$ & $1.3901(14)$ & $\mathrm{C} 26-\mathrm{C} 23$ & $1.5243(16)$ \\
$\mathrm{C} 20-\mathrm{N} 2$ & $1.4108(13)$ & $\mathrm{C} 26-\mathrm{N} 3$ & $1.4721(13)$ \\
$\mathrm{C} 11-\mathrm{H} 11$ & 0.9500 & $\mathrm{C} 3-\mathrm{H} 3$ & 0.9500 \\
$\mathrm{C} 11-\mathrm{C} 12$ & $1.3930(14)$ & $\mathrm{C} 3-\mathrm{C} 4$ & $1.3897(17)$ \\
$\mathrm{C} 11-\mathrm{C} 13$ & $1.3897(15)$ & $\mathrm{C} 24-\mathrm{H} 24 \mathrm{~A}$ & 0.9800 \\
$\mathrm{C} 18-\mathrm{H} 18$ & 0.9500 & $\mathrm{C} 24-\mathrm{H} 24 \mathrm{~B}$ & 0.9800 \\
$\mathrm{C} 18-\mathrm{C} 19$ & $1.3946(15)$ & $\mathrm{C} 24-\mathrm{H} 24 \mathrm{C}$ & 0.9800 \\
$\mathrm{C} 18-\mathrm{C} 17$ & $1.3836(14)$ & $\mathrm{C} 7-\mathrm{H} 7$ & 0.9500 \\
$\mathrm{C} 15-\mathrm{H} 15$ & 0.9500 & $\mathrm{C} 7-\mathrm{C} 6$ & $1.3903(18)$ \\
$\mathrm{C} 15-\mathrm{C} 16$ & $1.3807(15)$ & $\mathrm{C} 25-\mathrm{H} 25 \mathrm{~A}$ & 0.9800 \\
$\mathrm{C} 16-\mathrm{H} 16$ & 0.9500 & $\mathrm{C} 25-\mathrm{H} 25 \mathrm{~B}$ & 0.9800 \\
$\mathrm{C} 16-\mathrm{C} 17$ & $1.3953(15)$ & $\mathrm{C} 25-\mathrm{H} 25 \mathrm{C}$ & 0.9800 \\
$\mathrm{C} 22-\mathrm{C} 1$ & $1.5397(14)$ & $\mathrm{C} 4-\mathrm{H} 4$ & 0.9500 \\
$\mathrm{C} 22-\mathrm{N} 3$ & $1.3556(12)$ & $\mathrm{C} 4-\mathrm{C} 5$ & $1.3787(19)$ \\
$\mathrm{C} 22-\mathrm{N} 1$ & $1.2873(13)$ & $\mathrm{C} 21-\mathrm{H} 21 \mathrm{~A}$ & 0.9800 \\
$\mathrm{C} 10-\mathrm{H} 10$ & 0.9500 & $\mathrm{C} 21-\mathrm{H} 21 \mathrm{~B}$ & 0.9800 \\
$\mathrm{C} 10-\mathrm{C} 9$ & $1.3869(14)$ & $\mathrm{C} 21-\mathrm{H} 21 \mathrm{C}$ & 0.9800
\end{tabular}




\begin{tabular}{|c|c|c|c|}
\hline $\mathrm{C} 10-\mathrm{C} 13$ & $1.3906(15)$ & $\mathrm{C} 21-\mathrm{O} 2$ & $1.4220(15)$ \\
\hline $\mathrm{C} 12-\mathrm{H} 12$ & 0.9500 & $\mathrm{C} 14-\mathrm{H} 14 \mathrm{~A}$ & 0.9800 \\
\hline $\mathrm{C} 12-\mathrm{C} 8$ & $1.3930(15)$ & C14-H14B & 0.9800 \\
\hline C19-H19 & 0.9500 & $\mathrm{C} 14-\mathrm{H} 14 \mathrm{C}$ & 0.9800 \\
\hline $\mathrm{C} 8-\mathrm{C} 9$ & $1.4021(14)$ & $\mathrm{C} 14-\mathrm{O} 1$ & $1.4256(14)$ \\
\hline $\mathrm{C} 8-\mathrm{N} 1$ & $1.4120(13)$ & $\mathrm{C} 5-\mathrm{H} 5$ & 0.9500 \\
\hline $\mathrm{C} 1-\mathrm{H} 1$ & 1.0000 & $\mathrm{C} 5-\mathrm{C} 6$ & $1.385(2)$ \\
\hline $\mathrm{C} 1-\mathrm{C} 2$ & $1.5213(14)$ & $\mathrm{C} 23-\mathrm{H} 23 \mathrm{~A}$ & 0.9800 \\
\hline $\mathrm{C} 1-\mathrm{N} 2$ & $1.4643(13)$ & $\mathrm{C} 23-\mathrm{H} 23 \mathrm{~B}$ & 0.9800 \\
\hline $\mathrm{C} 17-\mathrm{O} 2$ & $1.3747(12)$ & $\mathrm{C} 23-\mathrm{H} 23 \mathrm{C}$ & 0.9800 \\
\hline C9- $\mathrm{H} 9$ & 0.9500 & $\mathrm{C} 6-\mathrm{H} 6$ & 0.9500 \\
\hline $\mathrm{C} 2-\mathrm{C} 3$ & $1.3868(15)$ & $\mathrm{O} 1-\mathrm{C} 13$ & $1.3794(12)$ \\
\hline $\mathrm{C} 2-\mathrm{C} 7$ & $1.3934(16)$ & $\mathrm{N} 2-\mathrm{H} 2$ & $0.887(15)$ \\
\hline $\mathrm{C} 26-\mathrm{C} 24$ & $1.5270(16)$ & $\mathrm{N} 3-\mathrm{H} 3 \mathrm{~A}$ & 0.8800 \\
\hline $\mathrm{C} 15-\mathrm{C} 20-\mathrm{N} 2$ & $119.16(9)$ & $\mathrm{C} 26-\mathrm{C} 24-\mathrm{H} 24 \mathrm{~A}$ & 109.5 \\
\hline $\mathrm{C} 19-\mathrm{C} 20-\mathrm{C} 15$ & $118.08(9)$ & $\mathrm{C} 26-\mathrm{C} 24-\mathrm{H} 24 \mathrm{~B}$ & 109.5 \\
\hline $\mathrm{C} 19-\mathrm{C} 20-\mathrm{N} 2$ & $122.74(9)$ & $\mathrm{C} 26-\mathrm{C} 24-\mathrm{H} 24 \mathrm{C}$ & 109.5 \\
\hline $\mathrm{C} 12-\mathrm{C} 11-\mathrm{H} 11$ & 120.3 & $\mathrm{H} 24 \mathrm{~A}-\mathrm{C} 24-\mathrm{H} 24 \mathrm{~B}$ & 109.5 \\
\hline $\mathrm{C} 13-\mathrm{C} 11-\mathrm{H} 11$ & 120.3 & $\mathrm{H} 24 \mathrm{~A}-\mathrm{C} 24-\mathrm{H} 24 \mathrm{C}$ & 109.5 \\
\hline $\mathrm{C} 13-\mathrm{C} 11-\mathrm{C} 12$ & $119.31(9)$ & $\mathrm{H} 24 \mathrm{~B}-\mathrm{C} 24-\mathrm{H} 24 \mathrm{C}$ & 109.5 \\
\hline $\mathrm{C} 19-\mathrm{C} 18-\mathrm{H} 18$ & 119.9 & $\mathrm{C} 2-\mathrm{C} 7-\mathrm{H} 7$ & 119.9 \\
\hline $\mathrm{C} 17-\mathrm{C} 18-\mathrm{H} 18$ & 119.9 & $\mathrm{C} 6-\mathrm{C} 7-\mathrm{C} 2$ & $120.22(12)$ \\
\hline $\mathrm{C} 17-\mathrm{C} 18-\mathrm{C} 19$ & $120.28(10)$ & $\mathrm{C} 6-\mathrm{C} 7-\mathrm{H} 7$ & 119.9 \\
\hline $\mathrm{C} 20-\mathrm{C} 15-\mathrm{H} 15$ & 119.5 & $\mathrm{C} 26-\mathrm{C} 25-\mathrm{H} 25 \mathrm{~A}$ & 109.5 \\
\hline $\mathrm{C} 16-\mathrm{C} 15-\mathrm{C} 20$ & $120.96(10)$ & $\mathrm{C} 26-\mathrm{C} 25-\mathrm{H} 25 \mathrm{~B}$ & 109.5 \\
\hline $\mathrm{C} 16-\mathrm{C} 15-\mathrm{H} 15$ & 119.5 & $\mathrm{C} 26-\mathrm{C} 25-\mathrm{H} 25 \mathrm{C}$ & 109.5 \\
\hline $\mathrm{C} 15-\mathrm{C} 16-\mathrm{H} 16$ & 119.8 & $\mathrm{H} 25 \mathrm{~A}-\mathrm{C} 25-\mathrm{H} 25 \mathrm{~B}$ & 109.5 \\
\hline $\mathrm{C} 15-\mathrm{C} 16-\mathrm{C} 17$ & $120.31(9)$ & $\mathrm{H} 25 \mathrm{~A}-\mathrm{C} 25-\mathrm{H} 25 \mathrm{C}$ & 109.5 \\
\hline $\mathrm{C} 17-\mathrm{C} 16-\mathrm{H} 16$ & 119.8 & $\mathrm{H} 25 \mathrm{~B}-\mathrm{C} 25-\mathrm{H} 25 \mathrm{C}$ & 109.5 \\
\hline $\mathrm{N} 3-\mathrm{C} 22-\mathrm{C} 1$ & $112.76(8)$ & $\mathrm{C} 3-\mathrm{C} 4-\mathrm{H} 4$ & 119.9 \\
\hline $\mathrm{N} 1-\mathrm{C} 22-\mathrm{C} 1$ & $124.98(9)$ & $\mathrm{C} 5-\mathrm{C} 4-\mathrm{C} 3$ & $120.11(12)$ \\
\hline $\mathrm{N} 1-\mathrm{C} 22-\mathrm{N} 3$ & $122.26(9)$ & $\mathrm{C} 5-\mathrm{C} 4-\mathrm{H} 4$ & 119.9 \\
\hline $\mathrm{C} 9-\mathrm{C} 10-\mathrm{H} 10$ & 119.9 & $\mathrm{H} 21 \mathrm{~A}-\mathrm{C} 21-\mathrm{H} 21 \mathrm{~B}$ & 109.5 \\
\hline $\mathrm{C} 9-\mathrm{C} 10-\mathrm{C} 13$ & $120.25(10)$ & $\mathrm{H} 21 \mathrm{~A}-\mathrm{C} 21-\mathrm{H} 21 \mathrm{C}$ & 109.5 \\
\hline $\mathrm{C} 13-\mathrm{C} 10-\mathrm{H} 10$ & 119.9 & $\mathrm{H} 21 \mathrm{~B}-\mathrm{C} 21-\mathrm{H} 21 \mathrm{C}$ & 109.5 \\
\hline $\mathrm{C} 11-\mathrm{C} 12-\mathrm{H} 12$ & 119.2 & $\mathrm{O} 2-\mathrm{C} 21-\mathrm{H} 21 \mathrm{~A}$ & 109.5 \\
\hline $\mathrm{C} 11-\mathrm{C} 12-\mathrm{C} 8$ & $121.63(10)$ & $\mathrm{O} 2-\mathrm{C} 21-\mathrm{H} 21 \mathrm{~B}$ & 109.5 \\
\hline $\mathrm{C} 8-\mathrm{C} 12-\mathrm{H} 12$ & 119.2 & $\mathrm{O} 2-\mathrm{C} 21-\mathrm{H} 21 \mathrm{C}$ & 109.5 \\
\hline $\mathrm{C} 20-\mathrm{C} 19-\mathrm{C} 18$ & $120.99(9)$ & $\mathrm{H} 14 \mathrm{~A}-\mathrm{C} 14-\mathrm{H} 14 \mathrm{~B}$ & 109.5 \\
\hline $\mathrm{C} 20-\mathrm{C} 19-\mathrm{H} 19$ & 119.5 & $\mathrm{H} 14 \mathrm{~A}-\mathrm{C} 14-\mathrm{H} 14 \mathrm{C}$ & 109.5 \\
\hline $\mathrm{C} 18-\mathrm{C} 19-\mathrm{H} 19$ & 119.5 & $\mathrm{H} 14 \mathrm{~B}-\mathrm{C} 14-\mathrm{H} 14 \mathrm{C}$ & 109.5 \\
\hline $\mathrm{C} 12-\mathrm{C} 8-\mathrm{C} 9$ & $118.01(9)$ & $\mathrm{O} 1-\mathrm{C} 14-\mathrm{H} 14 \mathrm{~A}$ & 109.5 \\
\hline $\mathrm{C} 12-\mathrm{C} 8-\mathrm{N} 1$ & $119.03(9)$ & $\mathrm{O} 1-\mathrm{C} 14-\mathrm{H} 14 \mathrm{~B}$ & 109.5 \\
\hline $\mathrm{C} 9-\mathrm{C} 8-\mathrm{N} 1$ & $122.89(9)$ & $\mathrm{O} 1-\mathrm{C} 14-\mathrm{H} 14 \mathrm{C}$ & 109.5 \\
\hline $\mathrm{C} 22-\mathrm{C} 1-\mathrm{H} 1$ & 108.1 & $\mathrm{C} 4-\mathrm{C} 5-\mathrm{H} 5$ & 120.2 \\
\hline $\mathrm{C} 2-\mathrm{C} 1-\mathrm{C} 22$ & $112.85(8)$ & $\mathrm{C} 4-\mathrm{C} 5-\mathrm{C} 6$ & $119.59(11)$ \\
\hline $\mathrm{C} 2-\mathrm{C} 1-\mathrm{H} 1$ & 108.1 & $\mathrm{C} 6-\mathrm{C} 5-\mathrm{H} 5$ & 120.2 \\
\hline
\end{tabular}




\begin{tabular}{|c|c|c|c|}
\hline $\mathrm{N} 2-\mathrm{C} 1-\mathrm{C} 22$ & $110.37(8)$ & $\mathrm{C} 26-\mathrm{C} 23-\mathrm{H} 23 \mathrm{~A}$ & 109.5 \\
\hline $\mathrm{N} 2-\mathrm{C} 1-\mathrm{H} 1$ & 108.1 & $\mathrm{C} 26-\mathrm{C} 23-\mathrm{H} 23 \mathrm{~B}$ & 109.5 \\
\hline $\mathrm{N} 2-\mathrm{C} 1-\mathrm{C} 2$ & $109.12(8)$ & $\mathrm{C} 26-\mathrm{C} 23-\mathrm{H} 23 \mathrm{C}$ & 109.5 \\
\hline $\mathrm{C} 18-\mathrm{C} 17-\mathrm{C} 16$ & $119.35(9)$ & $\mathrm{H} 23 \mathrm{~A}-\mathrm{C} 23-\mathrm{H} 23 \mathrm{~B}$ & 109.5 \\
\hline $\mathrm{O} 2-\mathrm{C} 17-\mathrm{C} 18$ & $123.94(10)$ & $\mathrm{H} 23 \mathrm{~A}-\mathrm{C} 23-\mathrm{H} 23 \mathrm{C}$ & 109.5 \\
\hline $\mathrm{O} 2-\mathrm{C} 17-\mathrm{C} 16$ & $116.71(9)$ & $\mathrm{H} 23 \mathrm{~B}-\mathrm{C} 23-\mathrm{H} 23 \mathrm{C}$ & 109.5 \\
\hline $\mathrm{C} 10-\mathrm{C} 9-\mathrm{C} 8$ & $120.74(10)$ & $\mathrm{C} 7-\mathrm{C} 6-\mathrm{H} 6$ & 119.8 \\
\hline $\mathrm{C} 10-\mathrm{C} 9-\mathrm{H} 9$ & 119.6 & $\mathrm{C} 5-\mathrm{C} 6-\mathrm{C} 7$ & $120.43(12)$ \\
\hline $\mathrm{C} 8-\mathrm{C} 9-\mathrm{H} 9$ & 119.6 & $\mathrm{C} 5-\mathrm{C} 6-\mathrm{H} 6$ & 119.8 \\
\hline $\mathrm{C} 3-\mathrm{C} 2-\mathrm{C} 1$ & $118.49(9)$ & $\mathrm{C} 17-\mathrm{O} 2-\mathrm{C} 21$ & $116.18(8)$ \\
\hline $\mathrm{C} 3-\mathrm{C} 2-\mathrm{C} 7$ & $118.73(10)$ & $\mathrm{C} 13-\mathrm{O} 1-\mathrm{C} 14$ & $117.04(9)$ \\
\hline $\mathrm{C} 7-\mathrm{C} 2-\mathrm{C} 1$ & $122.78(10)$ & $\mathrm{C} 20-\mathrm{N} 2-\mathrm{C} 1$ & $118.01(8)$ \\
\hline $\mathrm{C} 25-\mathrm{C} 26-\mathrm{C} 24$ & $109.45(9)$ & $\mathrm{C} 20-\mathrm{N} 2-\mathrm{H} 2$ & $112.8(9)$ \\
\hline $\mathrm{C} 23-\mathrm{C} 26-\mathrm{C} 24$ & $111.49(10)$ & $\mathrm{C} 1-\mathrm{N} 2-\mathrm{H} 2$ & $113.0(9)$ \\
\hline $\mathrm{C} 23-\mathrm{C} 26-\mathrm{C} 25$ & $109.80(10)$ & $\mathrm{C} 22-\mathrm{N} 3-\mathrm{C} 26$ & $127.34(9)$ \\
\hline $\mathrm{N} 3-\mathrm{C} 26-\mathrm{C} 24$ & $110.61(9)$ & $\mathrm{C} 22-\mathrm{N} 3-\mathrm{H} 3 \mathrm{~A}$ & 116.3 \\
\hline $\mathrm{N} 3-\mathrm{C} 26-\mathrm{C} 25$ & $105.50(9)$ & $\mathrm{C} 26-\mathrm{N} 3-\mathrm{H} 3 \mathrm{~A}$ & 116.3 \\
\hline $\mathrm{N} 3-\mathrm{C} 26-\mathrm{C} 23$ & $109.82(9)$ & $\mathrm{C} 22-\mathrm{N} 1-\mathrm{C} 8$ & $119.35(9)$ \\
\hline $\mathrm{C} 2-\mathrm{C} 3-\mathrm{H} 3$ & 119.6 & $\mathrm{C} 11-\mathrm{C} 13-\mathrm{C} 10$ & $119.96(9)$ \\
\hline $\mathrm{C} 2-\mathrm{C} 3-\mathrm{C} 4$ & $120.89(11)$ & $\mathrm{O} 1-\mathrm{C} 13-\mathrm{C} 11$ & $124.18(9)$ \\
\hline $\mathrm{C} 4-\mathrm{C} 3-\mathrm{H} 3$ & 119.6 & $\mathrm{O} 1-\mathrm{C} 13-\mathrm{C} 10$ & $115.85(9)$ \\
\hline $\mathrm{C} 20-\mathrm{C} 15-\mathrm{C} 16-\mathrm{C} 17$ & $-0.05(16)$ & $\mathrm{C} 9-\mathrm{C} 8-\mathrm{N} 1-\mathrm{C} 22$ & $-72.76(14)$ \\
\hline $\mathrm{C} 11-\mathrm{C} 12-\mathrm{C} 8-\mathrm{C} 9$ & $3.56(15)$ & $\mathrm{C} 2-\mathrm{C} 1-\mathrm{N} 2-\mathrm{C} 20$ & $-157.97(9)$ \\
\hline $\mathrm{C} 11-\mathrm{C} 12-\mathrm{C} 8-\mathrm{N} 1$ & $-179.35(9)$ & $\mathrm{C} 2-\mathrm{C} 3-\mathrm{C} 4-\mathrm{C} 5$ & $-0.11(19)$ \\
\hline $\mathrm{C} 18-\mathrm{C} 17-\mathrm{O} 2-\mathrm{C} 21$ & $4.27(15)$ & $\mathrm{C} 2-\mathrm{C} 7-\mathrm{C} 6-\mathrm{C} 5$ & $-0.7(2)$ \\
\hline $\mathrm{C} 15-\mathrm{C} 20-\mathrm{C} 19-\mathrm{C} 18$ & $-1.56(16)$ & $\mathrm{C} 3-\mathrm{C} 2-\mathrm{C} 7-\mathrm{C} 6$ & $-1.07(19)$ \\
\hline $\mathrm{C} 15-\mathrm{C} 20-\mathrm{N} 2-\mathrm{C} 1$ & $166.59(9)$ & $\mathrm{C} 3-\mathrm{C} 4-\mathrm{C} 5-\mathrm{C} 6$ & $-1.7(2)$ \\
\hline $\mathrm{C} 15-\mathrm{C} 16-\mathrm{C} 17-\mathrm{C} 18$ & $-1.01(16)$ & $\mathrm{C} 24-\mathrm{C} 26-\mathrm{N} 3-\mathrm{C} 22$ & $62.76(14)$ \\
\hline $\mathrm{C} 15-\mathrm{C} 16-\mathrm{C} 17-\mathrm{O} 2$ & $178.62(9)$ & $\mathrm{C} 7-\mathrm{C} 2-\mathrm{C} 3-\mathrm{C} 4$ & $1.49(17)$ \\
\hline $\mathrm{C} 16-\mathrm{C} 17-\mathrm{O} 2-\mathrm{C} 21$ & $-175.34(10)$ & $\mathrm{C} 25-\mathrm{C} 26-\mathrm{N} 3-\mathrm{C} 22$ & $-178.98(10)$ \\
\hline $\mathrm{C} 22-\mathrm{C} 1-\mathrm{C} 2-\mathrm{C} 3$ & $-145.84(10)$ & $\mathrm{C} 4-\mathrm{C} 5-\mathrm{C} 6-\mathrm{C} 7$ & $2.1(2)$ \\
\hline $\mathrm{C} 22-\mathrm{C} 1-\mathrm{C} 2-\mathrm{C} 7$ & $35.00(14)$ & $\mathrm{C} 14-\mathrm{O} 1-\mathrm{C} 13-\mathrm{C} 11$ & $3.97(15)$ \\
\hline $\mathrm{C} 22-\mathrm{C} 1-\mathrm{N} 2-\mathrm{C} 20$ & $77.49(11)$ & $\mathrm{C} 14-\mathrm{O} 1-\mathrm{C} 13-\mathrm{C} 10$ & $-176.82(10)$ \\
\hline $\mathrm{C} 12-\mathrm{C} 11-\mathrm{C} 13-\mathrm{C} 10$ & $-0.89(15)$ & $\mathrm{C} 23-\mathrm{C} 26-\mathrm{N} 3-\mathrm{C} 22$ & $-60.72(14)$ \\
\hline $\mathrm{C} 12-\mathrm{C} 11-\mathrm{C} 13-\mathrm{O} 1$ & $178.29(9)$ & $\mathrm{N} 2-\mathrm{C} 20-\mathrm{C} 15-\mathrm{C} 16$ & $179.73(9)$ \\
\hline $\mathrm{C} 12-\mathrm{C} 8-\mathrm{C} 9-\mathrm{C} 10$ & $-2.92(15)$ & $\mathrm{N} 2-\mathrm{C} 20-\mathrm{C} 19-\mathrm{C} 18$ & $-179.91(10)$ \\
\hline $\mathrm{C} 12-\mathrm{C} 8-\mathrm{N} 1-\mathrm{C} 22$ & $110.29(12)$ & $\mathrm{N} 2-\mathrm{C} 1-\mathrm{C} 2-\mathrm{C} 3$ & $91.07(11)$ \\
\hline $\mathrm{C} 19-\mathrm{C} 20-\mathrm{C} 15-\mathrm{C} 16$ & $1.32(15)$ & $\mathrm{N} 2-\mathrm{C} 1-\mathrm{C} 2-\mathrm{C} 7$ & $-88.08(12)$ \\
\hline $\mathrm{C} 19-\mathrm{C} 20-\mathrm{N} 2-\mathrm{C} 1$ & $-15.07(14)$ & $\mathrm{N} 3-\mathrm{C} 22-\mathrm{C} 1-\mathrm{C} 2$ & $-87.41(10)$ \\
\hline $\mathrm{C} 19-\mathrm{C} 18-\mathrm{C} 17-\mathrm{C} 16$ & $0.77(16)$ & $\mathrm{N} 3-\mathrm{C} 22-\mathrm{C} 1-\mathrm{N} 2$ & $34.97(11)$ \\
\hline $\mathrm{C} 19-\mathrm{C} 18-\mathrm{C} 17-\mathrm{O} 2$ & $-178.83(10)$ & $\mathrm{N} 3-\mathrm{C} 22-\mathrm{N} 1-\mathrm{C} 8$ & $-179.30(9)$ \\
\hline $\mathrm{C} 1-\mathrm{C} 22-\mathrm{N} 3-\mathrm{C} 26$ & $-176.49(9)$ & $\mathrm{N} 1-\mathrm{C} 22-\mathrm{C} 1-\mathrm{C} 2$ & $92.83(12)$ \\
\hline $\mathrm{C} 1-\mathrm{C} 22-\mathrm{N} 1-\mathrm{C} 8$ & $0.44(15)$ & $\mathrm{N} 1-\mathrm{C} 22-\mathrm{C} 1-\mathrm{N} 2$ & $-144.79(10)$ \\
\hline $\mathrm{C} 1-\mathrm{C} 2-\mathrm{C} 3-\mathrm{C} 4$ & $-177.70(10)$ & $\mathrm{N} 1-\mathrm{C} 22-\mathrm{N} 3-\mathrm{C} 26$ & $3.28(16)$ \\
\hline $\mathrm{C} 1-\mathrm{C} 2-\mathrm{C} 7-\mathrm{C} 6$ & $178.08(11)$ & $\mathrm{N} 1-\mathrm{C} 8-\mathrm{C} 9-\mathrm{C} 10$ & $-179.89(10)$ \\
\hline $\mathrm{C} 17-\mathrm{C} 18-\mathrm{C} 19-\mathrm{C} 20$ & $0.53(16)$ & $\mathrm{C} 13-\mathrm{C} 11-\mathrm{C} 12-\mathrm{C} 8$ & $-1.69(15)$ \\
\hline $\mathrm{C} 9-\mathrm{C} 10-\mathrm{C} 13-\mathrm{C} 11$ & $1.50(16)$ & $\mathrm{C} 13-\mathrm{C} 10-\mathrm{C} 9-\mathrm{C} 8$ & $0.45(16)$ \\
\hline
\end{tabular}


$\mathrm{C} 9-\mathrm{C} 10-\mathrm{C} 13-\mathrm{O} 1$

Hydrogen-bond geometry $\left(\AA,{ }^{\circ}\right)$

\begin{tabular}{lllll}
\hline$D-\mathrm{H} \cdots A$ & $D-\mathrm{H}$ & $\mathrm{H} \cdots A$ & $D \cdots A$ & $D-\mathrm{H} \cdots A$ \\
\hline $\mathrm{N} 2-\mathrm{H} 2 \cdots \mathrm{O}^{\mathrm{i}}$ & $0.887(15)$ & $2.328(15)$ & $3.1698(13)$ & $158.5(12)$ \\
\hline
\end{tabular}

Symmetry code: (i) $-x+1,-y+2,-z+2$. 